ARTICLE

https://doi.org/10.1038/s41467-019-11449-6

\title{
Location determination of metal nanoparticles relative to a metal-organic framework
}

\author{
Yu-Zhen Chen ${ }^{1,2}$, Bingchuan Gu ${ }^{3}$, Takeyuki Uchida4 ${ }^{4}$ Jiandang Liu ${ }^{3}$, Xianchun Liu ${ }^{5}$, Bang-Jiao Ye ${ }^{3}$, Qiang Xu (DD ${ }^{4} \&$ \\ Hai-Long Jiang (1) ${ }^{1}$
}

Metal nanoparticles (NPs) stabilized by metal-organic frameworks (MOFs) have been intensively studied in recent decades, while investigations on the location of guest metal NPs relative to host MOF particles remain challenging and very rare. In this work, we have developed several characterization techniques, including high-angle annular dark-field scanning transmission electron microscopy (HAADF-STEM) tomography, hyperpolarized ${ }^{129} \mathrm{Xe}$ NMR spectroscopy and positron annihilation spectroscopy (PAS), which are able to determine the specific location of metal NPs relative to the MOF particle. The fine PdCu NPs confined inside MIL-101 exhibit excellent catalytic activity, absolute selectivity and satisfied recyclability in the aerobic oxidation of benzyl alcohol in pure water. As far as we know, the determination for the location of metal NPs relative to MOF particles and pore structure information of metal NPs/MOF composites by ${ }^{129} \mathrm{Xe}$ NMR and PAS techniques has not yet been reported.

\footnotetext{
${ }^{1}$ Hefei National Laboratory for Physical Sciences at the Microscale, CAS Key Laboratory of Soft Matter Chemistry, Department of Chemistry, Collaborative Innovation Center of Suzhou Nano Science and Technology, University of Science and Technology of China, Hefei, Anhui 230026, P. R. China. ${ }^{2}$ College of Chemistry and Chemical Engineering, Qingdao University, Qingdao, Shandong 266071, P. R. China. ${ }^{3}$ State Key Laboratory of Particle Detection and Electronics, University of Science and Technology of China, Hefei, Anhui 230026, P. R. China. ${ }^{4}$ National Institute of Advanced Industrial Science and Technology (AIST), Ikeda, Osaka 563-8577, Japan. ${ }^{5}$ State Key Laboratory of Catalysis, Dalian Institute of Chemical Physics, Chinese Academy of Sciences, Zhongshan Road 457, Dalian 116023, P. R. China. Correspondence and requests for materials should be addressed to H.-L.J. (email: jianglab@ustc.edu.cn)
} 
$\mathrm{N}$ oble metal nanoparticles (NPs) have attracted intensive interest due to their particular electronic structure and physiochemical properties as well as potential applications in many fields, especially in catalysis ${ }^{1-7}$. However, the limited storage and the high cost of noble metal, such as Pt, evoke great efforts to develop their alternative catalysts. The combination of a transition metal with noble metal to form bimetallic NPs in an alloy or core-shell structure has become an effective strategy not only in decreasing the consumption of noble metals but also largely improving the catalytic performance because of synergistic effect between two metals ${ }^{8-14}$. For instance, the integration of $\mathrm{Pt}$ with $\mathrm{Cu}$ into bimetallic PtCu alloy NPs exhibits enhanced performance of $\mathrm{Pt}$ in electrooxidation of different alcohols ${ }^{12}$. In addition, the well-known size effect of metal nanoparticles (NPs) strongly affects their catalytic performance: small MNPs with large surface area are highly desirable. Unfortunately, these small MNPs with high surface energies readily aggregate to form larger MNPs with deteriorated catalytic activity ${ }^{15-17}$. Therefore, tremendous efforts have been dedicated to the exploitation of effective methods to stabilize MNPs, among which porous materials have been demonstrated to be very useful to restrict the growth of MNPs ${ }^{18-23}$.

Metal-organic frameworks (MOFs), emerging as a unique class of porous materials, have captured great research interest due to their highly ordered and tailorable pore structure, superhigh surface area, and potential applications in many fields, such as gas sorption and separation, sensor, drug delivery, etc. ${ }^{24-33}$. The permanent porosity of MOFs offers inherent conditions to confine small MNPs, and the crystalline porous structure of MOFs is expected to prevent the migration and aggregation of MNPs ${ }^{34-48}$. There have been quite a few reports on the stabilization of MNPs by MOFs based on different synthetic strategies, which can be mainly divided into three main approaches: (1) synthesis of surfactant-protected MNPs, followed by MOF epitaxial growth to give MNPs@MOF; ${ }^{34-36}$ (2) the assembly of surfactant-protected MNPs and MOF to give MNPs/MOF; ${ }^{37-39}$ (3) introduction of metal precursors into MOFs and subsequent reduction in situ to afford MNPs@MOF ${ }^{40-48}$. Among them, MNPs are well encapsulated inside and supported on MOFs, respectively, obtained by the first and second approaches, while the surfactantencapsulated MNPs in obtained nanocomposites are actually unfavorable as the surfactant possibly blocks the active sites to some extent. Delightedly, MNPs are surfactant free in MNPs/ MOF obtained by the last synthetic approach, and they exhibit considerable catalytic performances due to the fully accessible and clean surface of MNPs. Unfortunately, it remains a grand challenge to glean experimental evidences whether MNPs stay on or inside MOF particles.

Currently, the assertion for MNP location relative to MOF particles are mostly based on the sizes between MNPs and MOF pores, where smaller sizes of MNPs than MOF cavities are deemed to be embedded inside MOFs. Recently, size-selective catalysis has been employed as an important evidence to distinguish whether or not MNPs are encapsulated into MOF pores: small substrate has high conversion while large substrate cannot be accessible to MNPs inside a MOF thus not be reacted ${ }^{34-36}$. Although this solution is facile and the judgment is usually correct, it does not definitely reflect the real situation as the low catalytic efficiency of large substrates is sometimes ascribed to their inferior reaction kinetics to small ones. To provide credible information on the location of MNPs relative to MOF particles, we conducted X-ray absorption spectroscopy (XAS) to clearly demonstrate the incorporation of ultrafine $\mathrm{Au}_{2.5}$ clusters in a MOF with fluoro-coated channels ${ }^{42}$. However, synchrotron radiation source is not easily available, and MNPs are usually much larger than the clusters with several atoms only, even they are incorporated inside MOFs. XAS might not be the first choice for the location determination of MNPs relative to MOF particles. Therefore, to better understand the microstructure and structure-activity relationship of MNPs/MOF composites, it is highly desired to develop not only reliable but also readily available characterization techniques to determine the location of MNPs relative to MOF particles.

The ${ }^{129} \mathrm{Xe}$ NMR spectroscopy is specifically sensitive to the void spaces. In the past decades, it has become a well-established tool to investigate the pore structure information of porous materials, such as zeolite, porous carbon, porous polymer, mesoporous silica, etc. ${ }^{49-53}$, and complementary to conventional techniques that describe the solid part of materials. Accordingly, Xenon is also a sensitive probe to characterize the pores in MOFs by using either thermally polarized or hyperpolarized xenon ${ }^{54-57}$, although it has never been applied to the MNPs/MOF system yet thus far. Meanwhile, positron annihilation spectrometry (PAS) is one of the most powerful tools to detect vacancy-type defects, open volumes, and pores by measuring the lifetime of the positron ${ }^{58-61}$. The nondestructive and auto detective property makes it an effective method to study metals, semiconductors, and other porous materials ${ }^{60}$, Therefore, PAS should be also suitable to characterize the location of MNPs relative to MOF particle.

In this work, we have successfully incorporated monometallic $\mathrm{Pt}$ and bimetallic PtCu NPs into a MOF, with Pt and PtCu NP sizes of $1.5 \mathrm{~nm}$ and $1.7 \mathrm{~nm}$, respectively, via a double-solvent approach (DSA). Three advanced techniques, namely transmission electron microscopy (TEM) tomography, ${ }^{129} \mathrm{Xe}$ NMR spectroscopy, as well as PAS, have been cooperatively investigated the location of $\mathrm{Pt}$ and $\mathrm{PtCu}$ NPs relative to the host MOF particles. To the best of our knowledge, both ${ }^{129} \mathrm{Xe}$ NMR and positron annihilation techniques have not yet been employed to provide decisive evidences on whether MNPs are confined inside or located on MOFs. Significantly, thanks to the ultrafine PtCu NPs, the resultant PtCu@MOF exhibits excellent catalytic activity, selectivity, and recyclability toward the aerobic oxidation of benzyl alcohol in pure water.

\section{Results}

Synthesis and characterization of PtCu@MIL-101. The representative mesoporous MOF, Cr-MIL-101 with a molecular formula of $\mathrm{Cr}_{3} \mathrm{X}\left(\mathrm{H}_{2} \mathrm{O}\right)_{2} \mathrm{O}(\mathrm{BDC})_{3} \cdot \mathrm{nH}_{2} \mathrm{O} \quad(\mathrm{BDC}=$ benzene-1,4dicarboxylate, $\mathrm{X}=\mathrm{F}$ or $\mathrm{OH}, n \approx 25)^{62}$, was chosen as a host matrix to encapsulate MNPs due to its large specific surface area (BET, $\left.>3600 \mathrm{~m}^{2} \mathrm{~g}^{-1}\right)$, high-chemical stability, appropriate pores (2.9 and $3.4 \mathrm{~nm}$ ) accessible through two pore windows of $c a .1 .2$ and $1.6 \mathrm{~nm}$, respectively. The giant cages in MIL-101 are hydrophilic, which allow us to adopt a DSA approach to rationally incorporate the metal precursors into the cavities of MIL-10143,45. In brief, the precursor solution involving $\mathrm{H}_{2} \mathrm{PtCl}_{6} \cdot 6 \mathrm{H}_{2} \mathrm{O}$ and/or $\mathrm{Cu}\left(\mathrm{NO}_{3}\right)_{2} \cdot 3 \mathrm{H}_{2} \mathrm{O}$ with a volume slightly less than the MOF pore volume was absorbed into the pores of dehydrated MIL-101, thanks to the capillary force and hydrophilic interaction. Subsequently, the resultant metal precusors @MIL-101 composite was reduced by $20 \% \mathrm{H}_{2} / \mathrm{Ar}$ to afford tiny $\mathrm{Pd}$ and/or PdCu NPs (typically, $0.5 \mathrm{wt} \%$ metal loading otherwise mentioned) encapsulated in MIL-101.

No diffraction peak in the powder X-ray diffraction (PXRD) profiles can be assigned to $\mathrm{Pt}$ and $\mathrm{PtCu}$ NPs in the resultant nanocomposites (Supplementary Fig. 1), revealing that the obtained MNPs could be very small and/or low content of MNPs. The BET surface areas of as-synthesized MIL-101, Pt@MIL-101, $\mathrm{Pt}_{2} \mathrm{Cu}_{1} @ \mathrm{MIL}-101, \mathrm{Pt}_{1} \mathrm{Cu}_{1} @ \mathrm{MIL}-101, \mathrm{Pt}_{1} \mathrm{Cu}_{2} @ \mathrm{MIL}-101, \mathrm{Pt}_{1} \mathrm{Cu}_{3} @-$ MIL-101, $\mathrm{Pt}_{1} \mathrm{Cu}_{4} @$ MIL-101, and Cu@MIL-101 are 2904, 2645, $2545,2564,2329,2302,2254$, and $2479 \mathrm{~m}^{2} \mathrm{~g}^{-1}$, respectively, 

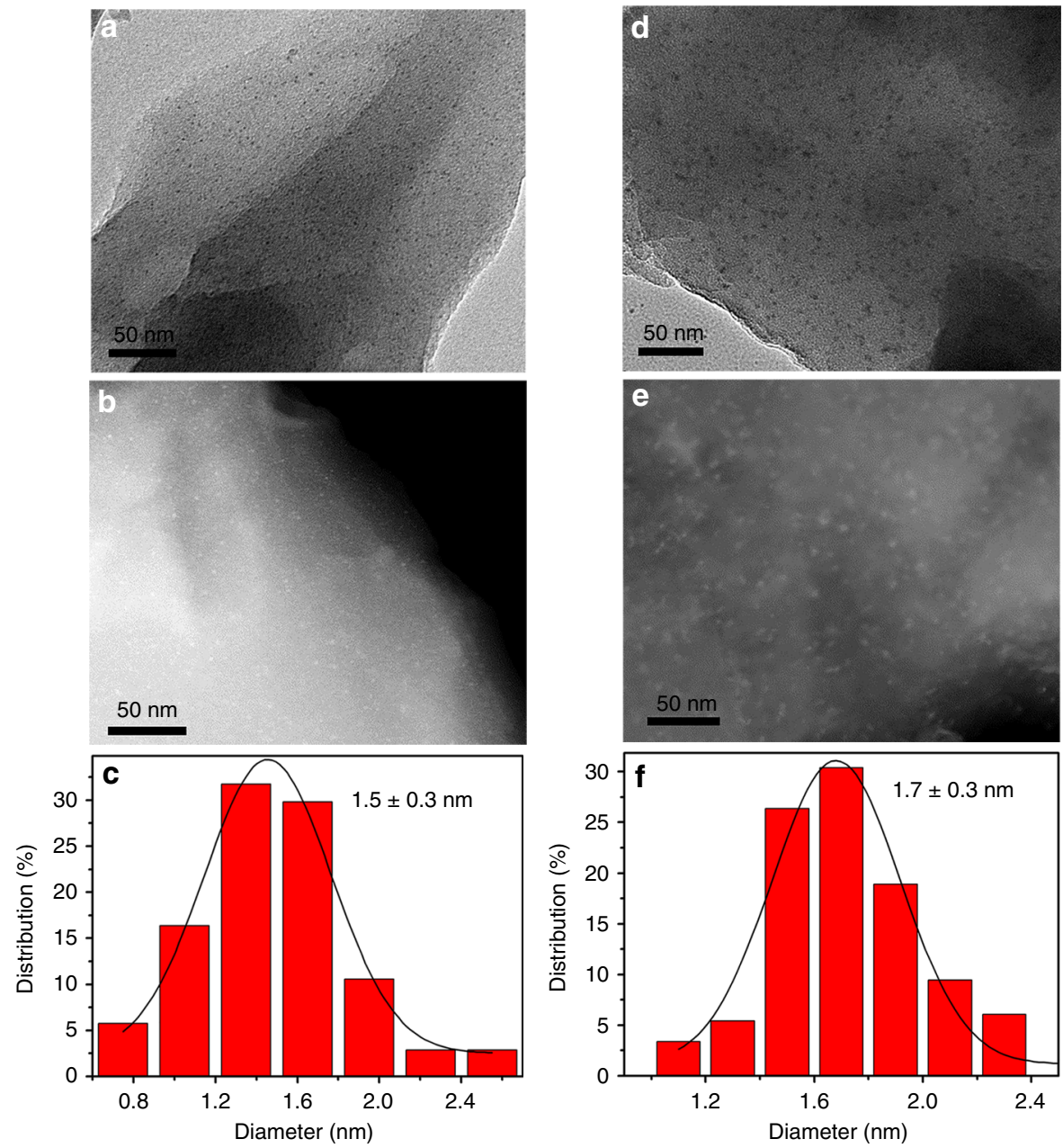

Fig. 1 TEM, HAADF-STEM images and size distribution. a TEM, b HAADF-STEM images, and $\mathbf{c}$ the corresponding size distribution of Pt NPs in Pt@MIL101. d TEM, e HAADF-STEM images, and $\mathbf{f}$ the corresponding size distribution of PtCu NPs in $\mathrm{Pt}_{1} \mathrm{Cu}_{2} @ M I L-101$

implying that the cavities of MOF are possibly occupied by highly dispersed MNPs (Supplementary Fig. 2). Inductively coupled plasma atomic emission spectrometry (ICP-AES) for several representative samples has confirmed that the actual contents of $\mathrm{Pt}$ and $\mathrm{Cu}$ are close to the nominal values, and the $\mathrm{Pt} / \mathrm{Cu}$ ratios almost agree the predesigned trend (Supplementary Table 1). An obvious positive shift in the binding energy of $\mathrm{Cu}$ $2 p_{3 / 2}$ can be observed for PtCu@MOF in reference to that of $\mathrm{Cu} @ \mathrm{MOF}$ from the X-ray photoelectron spectroscopy (XPS), which might be due to that the electrons on $\mathrm{Cu}$ surface flow into $\mathrm{Pt}$ considering the higher Fermi level of $\mathrm{Cu}$ than $\mathrm{Pt}$ (Supplementary Fig. 3$)^{9}$.

The TEM and high-angle annular dark-field scanning transmission electron microscopy (HAADF-STEM) images for Pt@MIL-101 (Fig. 1a，b) and PtCu@MIL-101 (Fig. 1d, e, $\mathrm{Pt}_{1} \mathrm{Cu}_{2} @$ MIL-101 as a representative hereafter unless otherwise stated) show Pt and PtCu NPs in ultrafine sizes of $\sim 1.5$ and $\sim 1.7 \mathrm{~nm}$ (Fig. 1c, f), respectively. As far as we know, this is among the smallest sizes of bimetallic NPs in the presence of surfactant protection thus far ${ }^{45}$. The smaller sizes of MNPs than the MOF pores hint the possible encapsulation of MNPs inside the giant cages. To confirm this, HAADF-STEM tomography was employed to examine the intrinsic morphology and spatial distribution of the nanocomposites ${ }^{37}$. The tomographic data were reconstructed to provide more detailed information for the $3 \mathrm{D}$ imaging of the real structure. A series of 3D HAADF-STEM images were taken for Pt@MIL-101 (Supplementary Fig. 4) and
$\mathrm{Pt}_{1} \mathrm{Cu}_{2} @ \mathrm{MIL}-101$ (Fig. 2) at consecutive tilt angles from $-62.6^{\circ}$ to $62.6^{\circ}$ with each $2^{\circ}$ tilt increment (videos 1 and 2 in the Supplementary Information). No agglomerated large Pt or $\mathrm{PtCu}$ NPs can be observed during the tilting process, revealing that MNPs are highly dispersed and embedded inside the MOF pores.

To further prove this, a series of tomographic slices unambiguously present the highly dispersed ultrafine PtCu NPs throughout the whole MIL-101 skeleton, demonstrating that almost all PtCu NPs are indeed trapped inside the MOF pores (Fig. 3, and video 3 in the Supplementary Information). The similar results gleaned from the tomographic slices of Pt@MIL101 also confirm the encapsulation of Pt NPs inside MIL-101 (Supplementary Fig. 5 and video 4 in the Supplementary Information). The above results clearly suggest the successful incorporation of $\mathrm{Pt}^{2+} / \mathrm{Cu}^{2+}$ into MOF cages via the strategy of DSA and the ideal confinement effect of MIL-101 for Pt and PtCu NPs.

Encouraged by the above results, hyperpolarized ${ }^{129} \mathrm{Xe}$ NMR technique was applied to illustrate the location of MNPs relative to MIL-101 particles. ${ }^{129} \mathrm{Xe}$, a noninvasive gas molecule, is able to mimic the behavior of guest atoms/molecules inside the pores, as the high polarizability of xenon electron cloud make it very sensitive to physical interactions with guest species in the pores, resulting in ${ }^{129} \mathrm{Xe}$ NMR chemical shift variation in reference to pristine porous materials (with empty pores). Therefore, the variable-temperature ${ }^{129} \mathrm{Xe}$ NMR spectroscopy has been recorded 


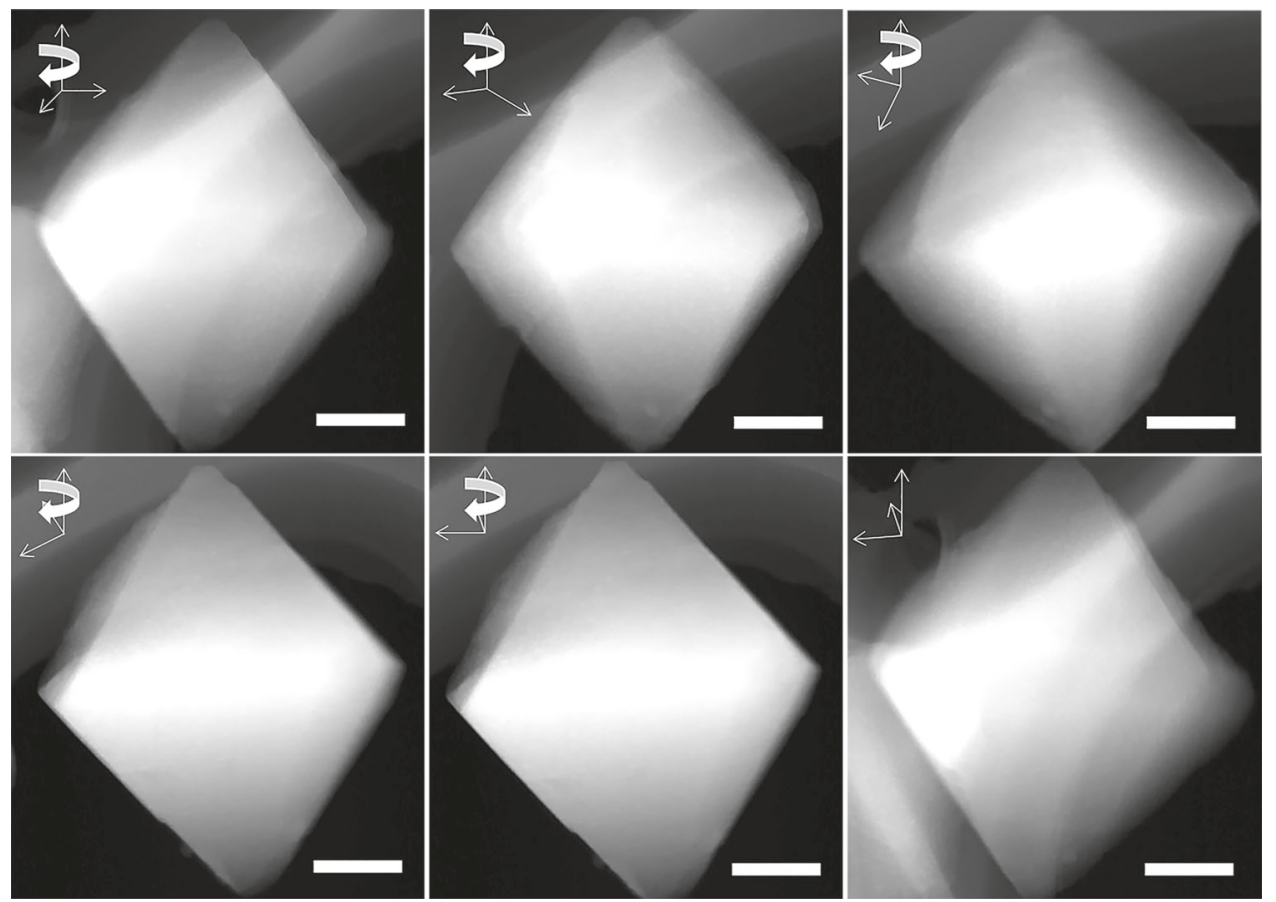

Fig. 2 HAADF-STEM images. The representative HAADF-STEM images captured from the video with a series of tilting angles for PtCu@MIL-101 sample taken with $2^{\circ}$ tilt increment step from $-62.6^{\circ}$ to $62.6^{\circ}$. Alpha tilt axis is parallel with $Z$ direction shown on the images. The scale bar on the images is $100 \mathrm{~nm}$

by using a continuous flow of hyperpolarized xenon adsorbed into MIL-101,Pt@MIL-101 and PtCu@MIL-101. Given that ${ }^{129} \mathrm{Xe}$ is chemically inert with a diameter of $0.44 \mathrm{~nm}$, much smaller than the pore windows and diameters of MIL-101, or even MIL-101 loaded with MNPs, it would easily go through the pores to access the guest MNPs. Prior to ${ }^{129} \mathrm{Xe} \mathrm{NMR}$ measurement, the samples were evacuated to remove residual guest molecules. Temperature-dependent ${ }^{129} \mathrm{Xe}$ NMR spectra for MIL-101, Pt@MIL-101, and PtCu@MIL-101 are shown in Fig.4a. The peaks at $0 \mathrm{ppm}$ are from the free ${ }^{129} \mathrm{Xe}$ gas, and the signals at lower field are originated from the absorbed Xe molecules inside MIL-101 pores. The signal intensity difference of the ${ }^{129} \mathrm{Xe}$ NMR signals for MIL-101, Pt@MIL-101, and PtCu@MIL-101 at a fixed temperature might be also related to the pore size and the stronger Xe adsorption at low temperatures. The chemical shifts of Xe molecules absorbed inside MIL-101 pores increase from $\sim 85$ to $\sim 200$ ppm along with decreased temperature from 293 to $193 \mathrm{~K}$ for all samples. This is a common trend in variabletemperature ${ }^{129} \mathrm{Xe}$ NMR spectra, mainly owing to the extended residence time of Xe on the internal surfaces and increased $\mathrm{Xe}-\mathrm{Xe}$ interactions at lower temperatures. At a fixed temperature (e.g., $293 \mathrm{~K}$ ) close to room temperature, the chemical shift (value) in hyperpolarized ${ }^{129} \mathrm{Xe}$ NMR spectra increases in the order of MIL$101<$ Pt@MIL-101<PtCu@MIL-101 due to their different sizes of the cavities (Fig. 4b). Given that the lower Xe concentration brings the greater chemical shift toward downfield, the data clearly suggest the cavity sizes in the order of MIL-101 > Pt@MIL$101>$ PtCu@MIL-101, in accordance with the occupied MOF pores by $\mathrm{Pt}$ and $\mathrm{PtCu}$ NPs, with slightly larger sizes of $\mathrm{PtCu}$ than Pt.

While some difference of chemical shift of ${ }^{129} \mathrm{Xe}$ adsorbed in the three samples can be observed at high temperature (close to room temperature), they are indeed similar at low temperatures (Fig. 4a, b). The result is mainly because of the very low metal loading $(<1 \mathrm{wt} \%)$ and small sizes of metal NPs in the composites, where only very limited MOF cavities are occupied. Moreover, along with decreasing temperatures, the higher Xe concentration in MIL-101 leads to enhanced interaction between $\mathrm{Xe}-\mathrm{Xe}$, in comparison with MNPs@MOF samples, which brings the greater chemical shift toward low field. This leads to the resultant close chemical shift in all three samples at low temperatures (Fig. 4a).

To further verify the applicability of ${ }^{129} \mathrm{Xe}$ NMR to identify the location of MNPs and augment the difference of chemical shift among these samples, 10 wt\% Pt@MIL-101, and 10 and 20 wt\% PtCu@MIL-101 have been deliberately synthesized for ${ }^{129} \mathrm{Xe}$ NMR measurement (Supplementary Fig. 6a, b). As expected, the chemical shift of ${ }^{129} \mathrm{Xe}$ adsorbed shows distinct difference and the chemical shift increases in the order of MIL-101 $<10 \mathrm{wt} \%$ Pt@MIL-101<10wt\% PtCu@MIL-101 due to their significantly different pore sizes. In addition, at a fixed temperature (e.g., 293 $\mathrm{K}$ ), hyperpolarized ${ }^{129} \mathrm{Xe}$ NMR spectra for MIL-101, and PtCu@MIL-101 with various PtCu NPs quality $(0.5,10$, and 20 wt $\%, \mathrm{n}_{\mathrm{Pt}} / \mathrm{n}_{\mathrm{Cu}}=1 / 2$ ) show that the Xe chemical shifts gradually increase along with the increased metal percentage from $0.5 \mathrm{wt} \%$ to $20 \mathrm{wt} \%$ of PtCu NPs in PtCu@MIL-101 (Fig. 4c). The results further demonstrate that the MNPs are mainly encapsulated in MOF pores.

To further verify the above results, positron annihilation (PA) technique has been adopted to evaluate the location of MNPs relative to the MOF particles, as positrons are very sensitive to the pores and/or defects on the atomic scale, which act as trap centers for positron. The positron lifetime spectra measured in nitrogen atmosphere (1 atm) with three lifetime components, $\tau 1(\mathrm{I} 1), \tau 2$ (I2), and $\tau 3$ (I3), by using LTv9 program ${ }^{63}$ for MIL-101 and $0.5 \mathrm{wt}$ \% PtCu@MIL-101 samples are shown in Fig. 4d. Their similar lifetime components are mainly due to the very low metal loading $(<1 \mathrm{wt} \%)$ and tiny MNPs in the sample. Therefore, 10 and $20 \mathrm{wt} \%$ Pt@MIL-101 as well as 10 and 20 wt\% PtCu@MIL-101 have been deliberately synthesized for PA measurement (Supplementary Fig. $6 c$, d, and Table 1). The lifetimes $(\tau 1, \tau 2$, and $\tau 3)$ are similar for three samples, while the intensities are quite different after loading $\mathrm{Pt}$ or $\mathrm{PtCu}$ NPs inside the MOF pores, especially for 

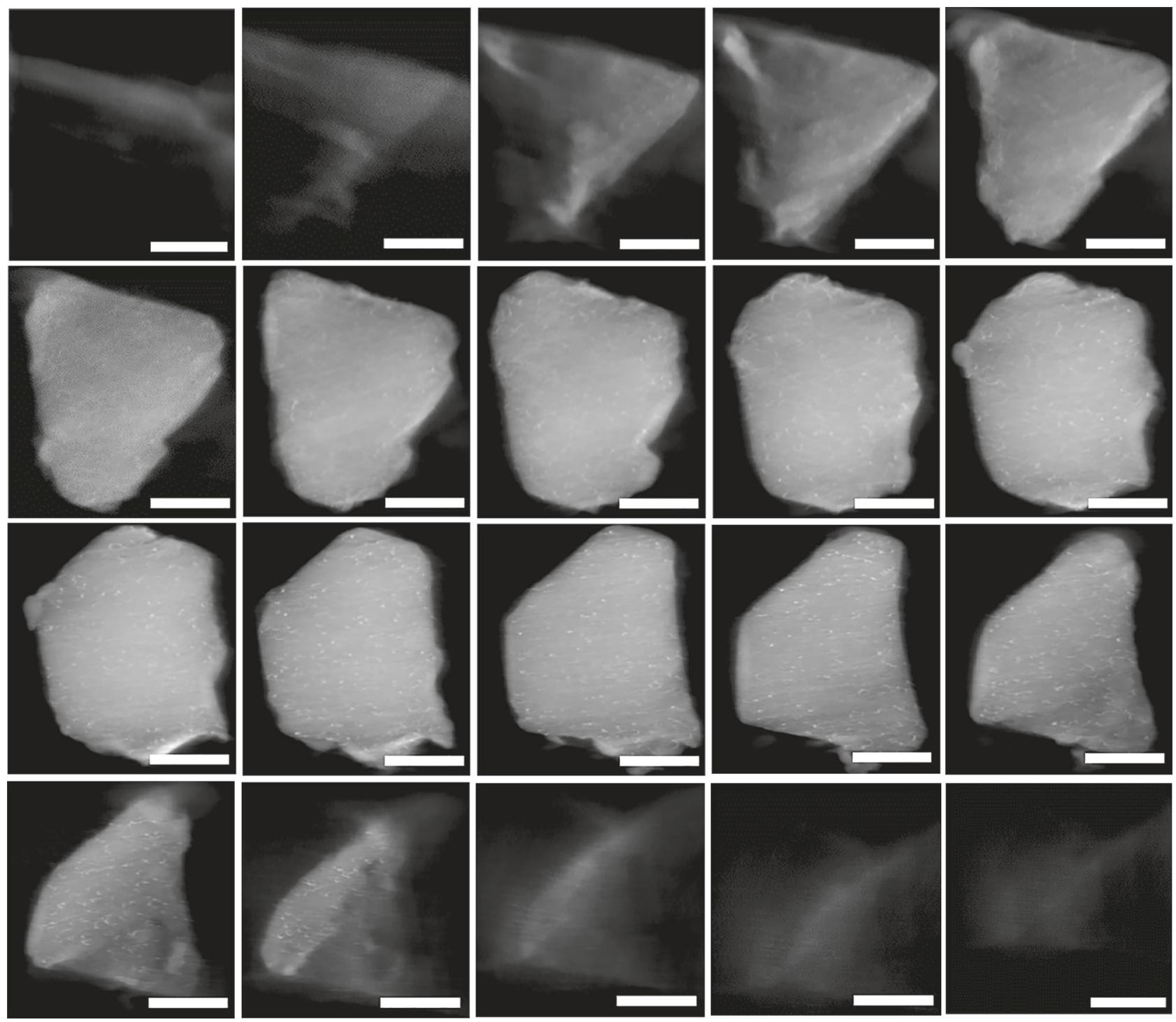

Fig. 3 Reconstructed slice images. Selected reconstructed slice images (observed in the order of left to right and top to down) throughout the entire $\mathrm{Pt}_{1} \mathrm{Cu}_{2} @ M I L-101$ skeleton. The scale bar on the images is roughly 200 nm

PtCu@MIL-101. Lifetimes shorter than 200 ps are usually found in the materials with high electron density, such as metal, alloy, or some of semiconductors ${ }^{64}$. Given that the electron density of MOF is relatively low, the positronium (Ps) is prone to form in the open volume regions. Therefore, the $\tau 1$ is most likely originated from the average of PA with free electrons and the spin singlet ( $\mathrm{p}$-Ps, mean lifetime of $125 \mathrm{ps}$ ) annihilation. The second lifetime ( $\tau 2)$ component at around 370 ps stems from the PA with the electrons in pores or defects. The intensities (I1, I2, and I3) for all samples are shown in Table 1 and Supplementary Fig. 7. The predominant intensity I2 (more than $70 \%$ ) implies that most of the positrons in the sample are trapped by the pores and/or defects in MOF. The longest lifetime of component $\tau 3$ longer than $2 \mathrm{~ns}$ represents the remained triplet states (o-Ps) formed at the surface or the interspace among the MOF particles. However, the intensity I3 is quite low (less than $1.5 \%$ ), which is probably because the o-Ps are quenched through pick-off or the other interactions due to the nitrogen molecules in the system. From the intensity comparison of three samples, after loading of $10 \mathrm{wt} \%$ PtCu NPs, I2 decreases from 88.6 to $76.4 \%$, and I1 increases from 10.9 to $22.1 \%$, which suggests that after loading of PtCu NPs, the free space in MOF pores becomes smaller and the long lifetime component decreases. Meanwhile, the PtCu NPs in MIL-101 might make o-Ps more easily quenched and quickly annihilated, which also leads to the increase of I1 and decrease of I2. In summary, the unchanged $\tau$ indicates that the main structure and the type of defects and pores (sensed by positrons) are remained before and after the NPs loading. However, the increase of I1 and decrease of $\mathrm{I} 2$ adequately demonstrates the incorporation of $\mathrm{Pt}$ or $\mathrm{PtCu}$ NPs inside the MOF pores. This can be explained by the obvious difference of the lifetime component for MIL-101, $10 \mathrm{wt}$ \% Pt@MIL-101, and 10wt\% PtCu@MIL-101 from the raw data (Supplementary Fig. 6c, d). There are quite a few possible reasons for the visible difference of I3 or $\tau 3$ between MIL-101 and $10 \mathrm{wt} \%$ PtCu@MOF (Table 1). Besides the MOF material itself, as no regular changes between tau3 and I3 observed in previous reports $^{64,65}$, the existence of $\mathrm{Cu}$ element would be responsible for the reduced lifetime. It is reported that the positrons prefer to annihilate on the $\mathrm{Cu}$ surface due to the lower positron affinity of $\mathrm{Cu}^{66,67}$. Moreover, the origin of the component $(\tau 3)$ is complicated: the o-Ps can be formed at the surface, or the interior, or even the interface of the sample, and surface morphology change and some uncertain factors such as background noise may also influence the o-Ps formation. As a control, 
a
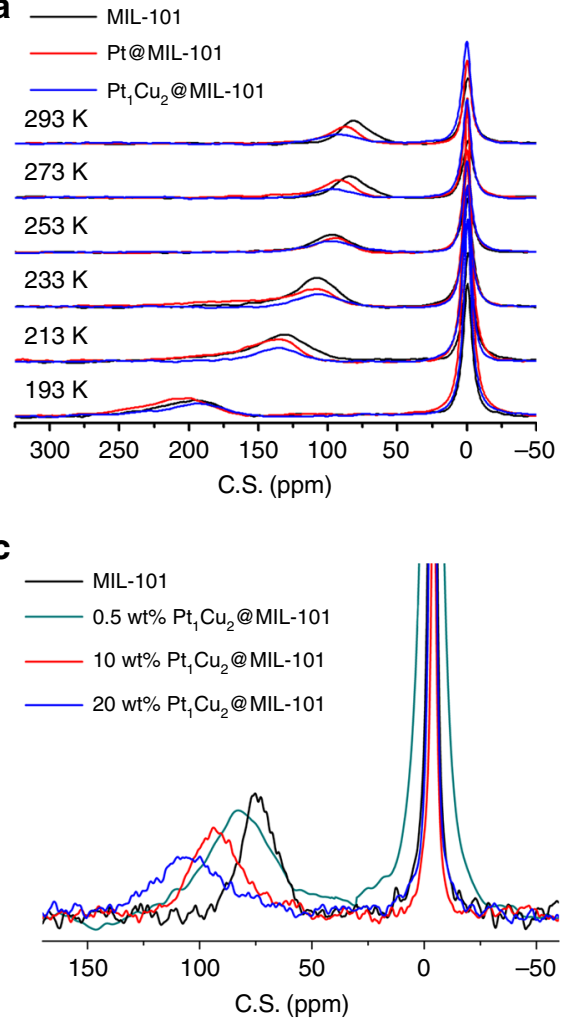

b

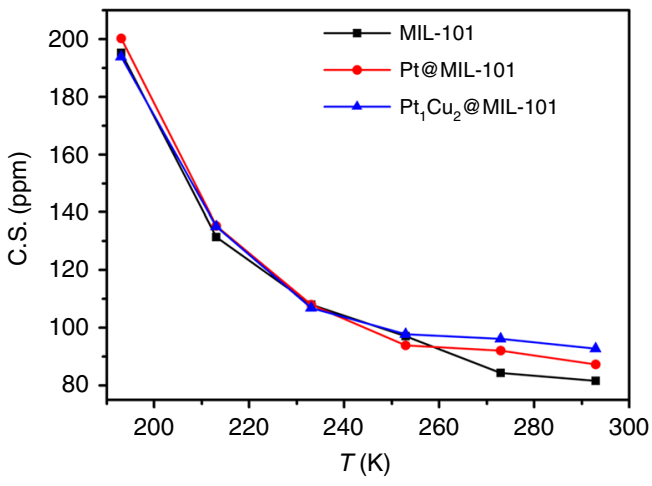

d

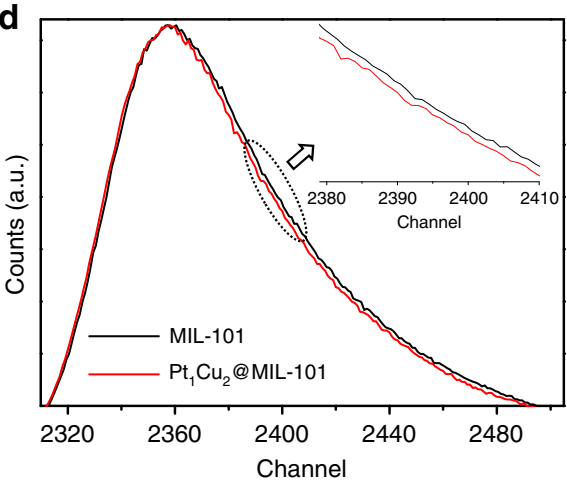

Fig. $4{ }^{129}$ Xe NMR and positron lifetime characterizations. a Temperature-dependent hyperpolarized ${ }^{129}$ Xe NMR spectra for MIL-101, Pt@MIL-101, and PtCu@MIL-101. The measurements were performed in the range of 193-293 K. b The 129 Xe chemical shift difference for MIL-101, Pt@MIL-101, and $\mathrm{Pt}_{1} \mathrm{Cu}_{2} @ \mathrm{MIL}-101$ at different temperatures. c Hyperpolarized ${ }^{129} \mathrm{Xe}$ NMR spectra performed at $293 \mathrm{~K}$ for MIL-101, and Pt $\mathrm{Cu}_{2} @ \mathrm{MIL}-101$ with various metal percentages. d Positron lifetime spectra for MIL-101 and 0.5 wt\% Pt $\mathrm{Cu}_{2} @$ MIL-101 based on positron annihilation experiments

Table 1 Positron lifetime parameters

\begin{tabular}{|c|c|c|c|c|c|c|}
\hline Sample & $\tau_{1}$ (ps) & $\tau_{2}$ (ps) & $\tau_{3}$ (ns) & $I_{1}(\%)$ & $I_{2}(\%)$ & $I_{3}(\%)$ \\
\hline MIL-101 & $192.0 \pm 1.5$ & $365.9 \pm 3.3$ & $2.65 \pm 0.20$ & $10.9 \pm 1.2$ & $88.6 \pm 2.0$ & $0.54 \pm 0.04$ \\
\hline 0.5wt\%PtCu@MOF & $188.0 \pm 2.4$ & $368.5 \pm 1.6$ & $2.59 \pm 0.12$ & $12.0 \pm 0.8$ & $86.4 \pm 1.8$ & $0.65 \pm 0.03$ \\
\hline 10 wt\% Pt@MOF & $180.1 \pm 9.6$ & $371.9 \pm 2.5$ & $2.45 \pm 0.16$ & $14.7 \pm 1.3$ & $84.5 \pm 1.3$ & $0.78 \pm 0.05$ \\
\hline 10 wt\% PtCu@MOF & $184.3 \pm 5.8$ & $374.5 \pm 1.7$ & $2.10 \pm 0.04$ & $22.1 \pm 0.8$ & $76.4 \pm 0.8$ & $1.49 \pm 0.03$ \\
\hline 20 wt\%Pt@MOF & $184.1 \pm 6.0$ & $373.0 \pm 2.5$ & $2.34 \pm 0.07$ & $23.4 \pm 0.5$ & $78.9 \pm 1.0$ & $1.06 \pm 0.05$ \\
\hline 20 wt\% PtCu@MOF & $186.0 \pm 5.8$ & $377.4 \pm 1.1$ & $2.05 \pm 0.06$ & $25.3 \pm 0.4$ & $73.4 \pm 1.2$ & $1.56 \pm 0.02$ \\
\hline MOF-5 & $215.8 \pm 6.3$ & $431.9 \pm 5.7$ & $2.90 \pm 0.19$ & $30.7 \pm 1.5$ & $58.2 \pm 1.4$ & $11.09 \pm 0.3$ \\
\hline
\end{tabular}

the PA spectrum of an as-synthesized MOF-5 has also been measured at room temperature in nitrogen atmosphere. The significant difference of the long-lifetime component between MIL-101 and MOF-5 can be obtained from the results (Table 1; Supplementary Fig. 8); moreover, the third component intensity (I3, $0.54 \%$ ) of MIL-101 is much lower than that (around 11\%) of MOF-5. These results suggest that the PAS are highly dependent on the specific MOF, and different MOF structures can present distinctly different results ${ }^{68-70}$.

Catalytic oxidation of alcohols by PtCu@MIL-101 catalysts. Encouraged by the perfect encapsulation of ultrafine $\mathrm{Pt}$ and $\mathrm{PtCu}$ NPs inside MIL-101, the selective aerobic oxidation of primary alcohols with molecular oxygen in water was investigated to evaluate the catalytic performance of the nanocomposites. Figure 5 summarizes the catalytic conversion and selectivity for the oxidation of benzyl alcohol over PtCu@MIL-101 catalysts with various $\mathrm{Pt} / \mathrm{Cu}$ molar ratios. The results indicate that $\mathrm{PtCu} @ \mathrm{MIL}-$
101 exhibit superior catalytic performance to monometallic Pt@MIL-101 and Cu@MIL-101 counterparts due to the synergistic effect between $\mathrm{Pt}$ and $\mathrm{Cu}$ species in the bimetallic catalysts. Evidently, the optimized PtCu@MIL-101 catalyst with $\mathrm{Pt} / \mathrm{Cu}$ molar ratio of 0.5 exhibits the highest conversion $(>99 \%)$ and selectivity (100\%) among all the catalysts with different $\mathrm{Pt} / \mathrm{Cu}$ ratios. To demonstrate the advantages of the confinement effect of MIL-101, $\mathrm{Pt}_{1} \mathrm{Cu}_{2} / \mathrm{MIL}-101$ with PtCu NPs mainly located on the surface of MIL-101 prepared by incipient wetness impregnation for comparison, exhibits lower yield (85\%) to the target product under similar conductions, possibly due to the aggregated PtCu NPs with the lack of sufficient confinement effect.

Recycling test and oxidation of various aromatic alcohols. No significant loss of crystallinity for the MOF and no identifiable peak for PtCu NPs can be observed in the PXRD pattern of PtCu@MIL-101 after reaction, indicating its high stability and good confinement of MIL-101 (Fig. 6b). Moreover, the catalytic 
activity and selectivity over $\mathrm{Pt}_{1} \mathrm{Cu}_{2} @ \mathrm{MIL}-101$ almost remains preserved during three cycles (Supplementary Fig. 9). No significant aggregation occurs to $\mathrm{PtCu}$ NPs even after three times of use from the representative TEM image for $\mathrm{Pt}_{1} \mathrm{Cu}_{2} @ \mathrm{MIL}-101$ (Fig. 6a). However, the contrast sample $\mathrm{Pt}_{1} \mathrm{Cu}_{2} / \mathrm{MIL}-101$, with $\mathrm{PtCu}$ NPs supported on the external surface of the MOF, presents apparent aggregation of PtCu NPs from 3.1 to $~ 7.4 \mathrm{~nm}$ after three cycles under identical reaction conditions, causing the fading of recycling performance during stability test (Supplementary Figs. 10,11). These results unambiguously suggest that the superb confinement effect offered by MIL-101 plays a vital role in preventing from aggregation and the excellent catalytic performance of tiny PtCu NPs, highlighting the importance of the MNP location relative to MOF particles. Encouraged by the outstanding catalytic performance of PtCu@MIL-101 in the oxidation of benzyl alcohol, we have extended this reaction to more substrates. To our delight, diverse substituted benzyl alcohols and heteroaromatic alcohols were completely oxidized within $5 \mathrm{~h}$ to corresponding aldehydes with almost absolute selectivity (Table 2), well demonstrating the general applicability of PtCu@MIL-101 catalysts. To further verify the good conversion and selectivity of the catalyst, the reaction with increased amount of substrate has been examined (Table 2, entry 12). The oxidation of alcohol can be almost completed with $93 \%$ conversion and $98 \%$ selectivity to benzaldehyde within $12 \mathrm{~h}$, indicating the great adaptability of

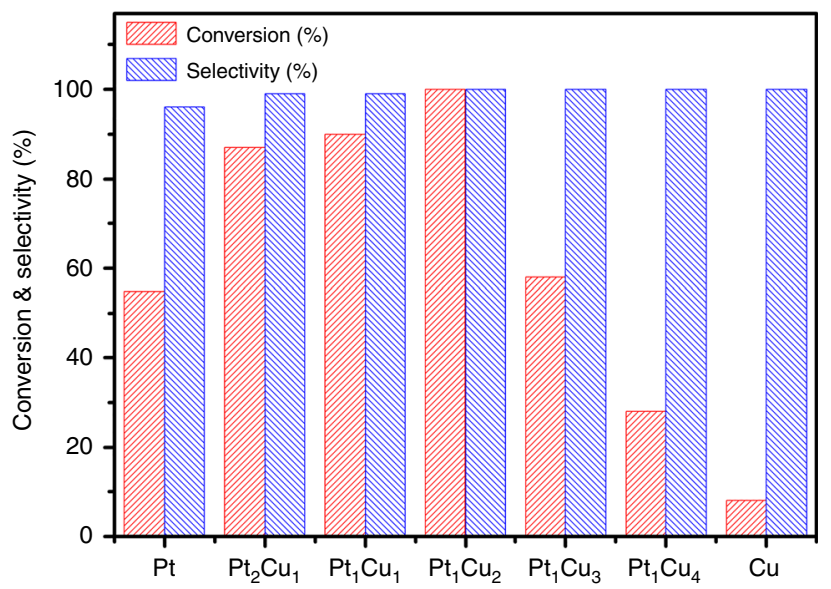

Fig. 5 Oxidation of benzyl alcohol over PtCu@MIL-101 catalysts. Conversion and selectivity of catalytic oxidation of benzyl alcohol over PtCu@MIL-101 catalysts with different Pt/Cu molar ratios. Reaction conditions: catalyst (100 mg), alcohol $(0.2 \mathrm{mmol}), \mathrm{H}_{2} \mathrm{O}(5 \mathrm{~mL}), \mathrm{O}_{2}$ $(0.5 \mathrm{MPa}), 373 \mathrm{~K}$, reaction time for each run is $5 \mathrm{~h}$
$\mathrm{Pt}_{1} \mathrm{Cu}_{2} @ M I L-101$. Moreover, no detectable benzoic acid product even after extended reaction time indicates the excellent selectivity toward aldehyde by $\mathrm{Pt}_{1} \mathrm{Cu}_{2} @ \mathrm{MIL}-101$.

Size-selective catalysis over $\mathrm{Pt}_{1} \mathrm{Cu}_{2} @$ MIL-101 and $\mathrm{Pt}_{1} \mathrm{Cu}_{2} / \mathrm{C}$. To further demonstrate the intact MIL-101 structure after loading MNPs, size-selective catalysis over MNPs@MIL-101 has been conducted. To this end, styrene (molecular size $\sim 3.5 \AA$ ), cyclooctene (molecular size $\sim 5.7 \AA$ ), and tetraphenyl ethylene (11.6 $\AA$ ) were chosen for hydrogenation reactions (Supplementary Table 2). For comparison, we prepared the $\mathrm{Pt}_{1} \mathrm{Cu}_{2} /$ graphite carbon (denoted $\mathrm{Pt}_{1} \mathrm{Cu}_{2} / \mathrm{C}$ ) as a control, where $\mathrm{PtCu}$ NPs were located on the external surface of graphite carbon. Both $\mathrm{Pt}_{1} \mathrm{Cu}_{2} @ M I L-101$ and $\mathrm{Pt}_{1} \mathrm{Cu}_{2} / \mathrm{C}$ showed $>99 \%$ conversion of styrene after $10 \mathrm{~min}$ reaction at room temperature, indicating that MIL-101 shell almost has no influence on the diffusion of styrene. For cyclooctene with a larger molecular size, $\mathrm{Pt}_{1} \mathrm{Cu}_{2} @ \mathrm{MIL}-101$ gives 29 and $70 \%$ conversions while $\mathrm{Pt}_{1} \mathrm{Cu}_{2} / \mathrm{C}$ achieves 56 and $>99 \%$ conversions, respectively, after 30 - and 60 -min reaction. More strikingly, $\mathrm{Pt}_{1} \mathrm{Cu}_{2} / \mathrm{C}$ presented significant activity $(34 \%$ conversion) for tetraphenyl ethylene hydrogenation, in stark contrast to the negligible activity ( $<5 \%)$ of $\mathrm{Pt}_{1} \mathrm{Cu}_{2} @ \mathrm{MIL}-101$, in $60 \mathrm{~min}$, because of the difficult substrate diffusion through MOF pore windows. Given the remarkable size-selective catalytic behavior of $\mathrm{Pt}_{1} \mathrm{Cu}_{2} @ \mathrm{MIL}-101$ and activity comparison with $\mathrm{Pt}_{1} \mathrm{Cu}_{2} / \mathrm{C}$ described above, the structure of MIL-101 should be well retained during loading MNPs. In addition, the results of size-selective catalysis once again demonstrate that the MNPs are mainly encapsulated inside MOF pores.

\section{Discussion}

In summary, we have successfully clarified the specific location of MNPs relative to MOF particles by several characterization techniques, including HAADF-STEM tomography with the slice technology, hyperpolarized ${ }^{129} \mathrm{Xe}$ NMR spectroscopy, and positron annihilation spectroscopy (PAS). To the best of our knowledge, this is the first time to determine MNPs location relative to MOF particles as well as the pore structure information of $\mathrm{MNP} / \mathrm{MOF}$ by ${ }^{129} \mathrm{Xe}$ NMR and PAS techniques. The development of the current three reliable techniques would terminate the long-term controversy on the location of MNPs relative to MOFs, which remains to be a grand challenge and significantly affects the catalytic performance ${ }^{47}$. In addition, the obtained ultrafine PtCu alloy NPs are relatively low cost and exhibit superior catalytic performance to the monometallic counterparts owing to the synergetic effect between $\mathrm{Pt}$ and $\mathrm{Cu}$, in the aerobic oxidation of primary alcohols using molecular $\mathrm{O}_{2}$ in pure water. The optimal $\mathrm{Pt}_{1} \mathrm{Cu}_{2} @ \mathrm{MIL}-101$ catalyst not only
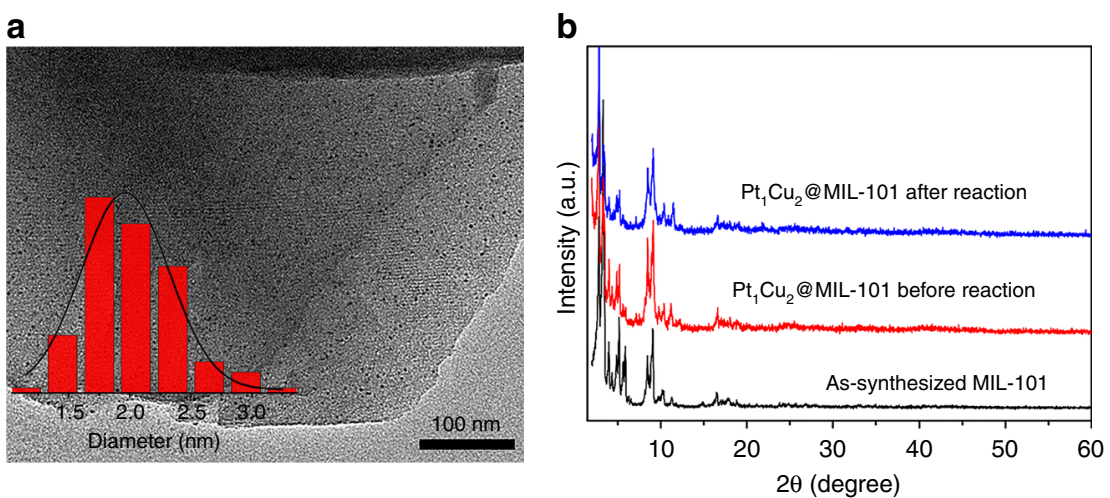

Fig. 6 TEM image and PXRD patterns. a The TEM image and (inset) the corresponding size distribution of PtCu NPs in Pt ${ }_{1} \mathrm{Cu}_{2} @ M I L-101$ after three catalytic cycles. b Wide-angle PXRD patterns of MIL-101 and $\mathrm{Pt}_{1} \mathrm{Cu}_{2} @ \mathrm{MIL}-101$ before and after catalytic reaction 
Table 2 Selective aerobic oxidation of various aromatic alcohols over $\mathrm{Pt}_{1} \mathrm{Cu}_{2} @ M I L-101^{\mathrm{a}}$

Canversion (\%) $)^{\mathrm{b}}$

aReaction conditions: catalyst $(100 \mathrm{mg})$, alcohol $(0.2 \mathrm{mmol}), \mathrm{H}_{2} \mathrm{O}(5 \mathrm{~mL}), \mathrm{O}_{2}(0.5 \mathrm{MPa}), 373 \mathrm{~K}$, reaction time is $5 \mathrm{~h}$. bYield of aldehyde was analyzed by $\mathrm{GC}$, and $n$-dodecane was used as the internal standard. cSubstrate $(0.1 \mathrm{mmol})$. dSubstrate $(0.5 \mathrm{mmol}$, reaction time is $12 \mathrm{~h})$

exhibits excellent catalytic activity and absolute selectivity to the corresponding aldehydes but also performs great recyclability by taking advantages of the confinement effect of MIL-101 for $\mathrm{PtCu} \mathrm{NPs}$ even under moderately high reaction temperature and pressure. We believe that this work would pave the way to the detection of guest species location relative to diverse host porous materials and thus greatly promote the development of related host-guest nanocomposites for applications, especially in catalysis.

\section{Methods}

Materials and equipment. All chemicals were from commercial suppliers without further purification unless otherwise mentioned. Hexachloroplatinic acid hexahydrate $\left(\mathrm{H}_{2} \mathrm{PtCl}_{6} \cdot 6 \mathrm{H}_{2} \mathrm{O}\right)$ was from Beijing Hwrk Chem Co., Ltd. Chromium(III) nitrate nonahydrate $\left(\mathrm{Cr}\left(\mathrm{NO}_{3}\right)_{3} \cdot 9 \mathrm{H}_{2} \mathrm{O}, 99 \%\right)$ was from Sigma-Aldrich. Powder Xray diffraction patterns (PXRD) were conducted on a Japan Rigaku SmartLab ${ }^{\mathrm{TM}}$ rotation anode X-ray diffractometer equipped or Holland X-Pert PRO fixed anode $\mathrm{X}$-ray diffractometer equipped with graphite monochromatized $\mathrm{Cu}$ Ka radiation $(\lambda=1.54178 \AA)$. The contents of $\mathrm{Pt}$ and $\mathrm{Cu}$ in the nanocomposites were quantified by an Optima $7300 \mathrm{DV}$ inductively coupled plasma atomic emission spectrometer
(ICP-AES). The X-ray photoelectron spectroscopy (XPS) measurements were carried out using an ESCALAB 250 high-performance electron spectrometer using monochromatized $\mathrm{Al} \mathrm{Ka}(\mathrm{h} v=1486.7 \mathrm{eV})$ as the excitation source. The size, morphology, and microstructure of PtCu@MIL-101 samples were preliminarily investigated by using the transmission electron microscopy (TEM) and reconstructed slices from high-angle annular dark-field scanning transmission electron microscopy (HAADF-STEM) on JEOL-2010 and JEOL-2100F instruments with an electron acceleration energy of $200 \mathrm{kV}$. Nitrogen sorption isotherms were measured by using an automatic volumetric adsorption equipment (Micrometritics, ASAP 2020). Prior to nitrogen sorption measurements, the as-synthesized samples were sequentially activated in water, $\mathrm{EtOH}$, and $\mathrm{NH}_{4} \mathrm{Cl}$ aqueous solution, finally dried in vacuum at $60^{\circ} \mathrm{C}$. Following that, the product was dried again by using the outgas function for $12 \mathrm{~h}$ at $150{ }^{\circ} \mathrm{C}$. Catalytic reaction products were analyzed and identified by gas chromatography (GC, Shimadzu 2010 Plus with a $0.25 \mathrm{~mm} \times 30 \mathrm{~m}$ Rtx -5 capillary column).

Tomography and slice techniques. To further verify whether NPs are really inside the MOF, a series of reconstructed slices were taken with $2^{\circ}$ step as tilting sample stage from $-62.6^{\circ}$ to $62.6^{\circ}$ by using FEI Tecnai G2 F20 TEM, operated at $200 \mathrm{keV}$. Computational reconstruction and visualization were conducted using the INSPECT3D and the AVIZO software package, respectively.

Hyperpolarized ${ }^{129} \mathrm{Xe}$ NMR measurements. The ${ }^{129} \mathrm{Xe}$ NMR experiments were carried out at $110.6 \mathrm{MHz}$ on the Varian Infinity-plus 400 spectrometer using a $7.5 \mathrm{~mm}$ probe. Prior to each experiment, the samples were subjected to dehydration at $423 \mathrm{~K}$ under vacuum $\left(<10^{-5}\right.$ Torr $)$ for overnight. A flow of $1 \% \mathrm{Xe}, 1 \% \mathrm{~N}_{2}$, and $98 \% \mathrm{He}$ gas mixture was delivered at the rate of $150 \mathrm{~cm}^{3} \mathrm{~min}^{-1}$ to the sample in detection region via plastic tubing. Variable-temperature NMR measurements were performed in the range of 193-293 K. All one-dimensional spectra were acquired with $3.0 \mu \mathrm{s} \pi / 2$ pulse, $100-200$ scans, and $2 \mathrm{~s}$ recycle delay. The chemical shifts were referenced to the signal of xenon gas. Although this line was temperature dependent, its chemical shift variation would not be more than $1 \mathrm{ppm}$ in the whole range of measurements because of the very low concentration of xenon.

Positron annihilation measurement. The MIL-101, Pt@MIL-101, $\mathrm{Pt}_{1} \mathrm{Cu}_{2} @ \mathrm{MIL}$ 101 , and MOF-5 samples were pressed under a static pressure of about $5 \mathrm{MPa}$ for about $3 \mathrm{~min}$ at room temperature to get disk shape pellets with a diameter of 10 $\mathrm{mm}$ and a thickness of $1 \mathrm{~mm}$. Positron lifetime measurements were conducted using an ORTEC fast-fast coincidence system in nitrogen atmosphere. The time resolution of the system is about 240 ps in full width at half maximum (FWHM) Each spectrum was collected with a total count of $2 \times 106$. A $20 \mu \mathrm{Ci}$ source of ${ }^{22} \mathrm{Na}$ was sandwiched between two identical sample pellets. Some notes should be pointed out with regards to the measurement: (i) the samples must be adequately dried overnight at $160^{\circ} \mathrm{C}$ under vacuum and then should be always protected in nitrogen environment preventing from water absorption until test. (ii) Laminating the sample is a critical step. Generally, the sample should be pressed with a static pressure of about $5 \mathrm{MPa}$ for about $3 \mathrm{~min}$ at room temperature to get disk shape pellets with a diameter of $10 \mathrm{~mm}$ (the two pieces must be kept equal mass and volume). (iii) The thickness of the sheet sample should be neither too thick nor too thin. In this work, $80 \mathrm{mg}$ of sample with a thickness of $1 \mathrm{~mm}$ was just right. (iii) The entire tableting process should be rapid, and the samples were tested immediately after the pressing.

Preparation of MIL-101. Typically, a mixture of $332 \mathrm{mg}$ of terephthalic acid with $800 \mathrm{mg}$ of $\mathrm{Cr}\left(\mathrm{NO}_{3}\right)_{3} \cdot 9 \mathrm{H}_{2} \mathrm{O}$ in the presence of aqueous $\mathrm{HF}(0.4 \mathrm{~mL}, 2.0 \mathrm{mmol})$ and deionized water $(9.5 \mathrm{~mL})$ was reacted at $200^{\circ} \mathrm{C}$ for $8 \mathrm{~h}$. The as-synthesized MIL101 was purified in water at reflux temperature for $24 \mathrm{~h}$ followed by in ethanol at $100^{\circ} \mathrm{C}$ for $24 \mathrm{~h}$ for twice and washed with hot ethanol, and was further purified by $\mathrm{NH}_{4} \mathrm{~F}$ solution. The result sample was finally dried overnight at $160^{\circ} \mathrm{C}$ under vacuum for further use.

Preparation of MOF-5. Typically, terephthalic acid $(0.507 \mathrm{~g})$ and triethylamine $(0.85 \mathrm{~mL})$ were dissolved in $40 \mathrm{~mL}$ of N,N-dimethylformamide (DMF); $\mathrm{Zn}$ $(\mathrm{OAc})_{2} \cdot 2 \mathrm{H}_{2} \mathrm{O}(1.7 \mathrm{~g})$ was dissolved in DMF $(50 \mathrm{~mL})$. The $\mathrm{Zn}$-based solution was added into the ligand solution under stirring over $15 \mathrm{~min}$, forming a precipitate, and the mixture was kept stirring for over $2.5 \mathrm{~h}$. The precipitate was purified by $\mathrm{DMF}$, and then was separated by centrifugation followed by being immersed in $\mathrm{CHCl}_{3}$. The resultant MOF-5 powder was dried overnight at $120^{\circ} \mathrm{C}$ under vacuum condition.

Preparation of Pt@MIL-101, Cu@MIL-101, and PtCu@MIL-101. Typically, $200 \mathrm{mg}$ of activated MIL-101 was suspended in $40 \mathrm{~mL}$ of a hydrophobic solvent of dry $\mathrm{n}$-hexane, and the mixture was sonicated for around $20 \mathrm{~min}$ until it became homogeneous. After the mixture was stirred for a certain time, $0.30 \mathrm{~mL}$ of hydrophilic aqueous $\mathrm{H}_{2} \mathrm{PtCl}_{6} \cdot 6 \mathrm{H}_{2} \mathrm{O}$ and/or $\mathrm{Cu}\left(\mathrm{NO}_{3}\right)_{2}$ solution with the desired concentration were added dropwise with a syringe pump over a period of $20 \mathrm{~min}$ with constant vigorous stirring. Subsequently, the resultant solution was continuously stirred for $3 \mathrm{~h}$. The harvested sample was further dried followed by 
treating in a stream of $20 \% \mathrm{H}_{2} / \mathrm{Ar}$ at $200^{\circ} \mathrm{C}$ for $4 \mathrm{~h}$ to yield Pt@MIL-101, Cu@MIL101, and PtCu@MIL-101. The Pt/Cu molar ratios were changed (2, 1, 0.5, 0.33 , and 0.25 ) to optimize the activity of the resultant catalysts, while the total content of both metals was fixed to be $0.5 \mathrm{wt} \%$. The successful preparation of ultrafine MNPs is affected by the suitable MIL-101 host, the metal precursor introduction technique (DSA) as well as the reduction method.

Preparation of $\mathbf{P t}_{1} \mathbf{C u}_{2} / \mathbf{M I L}-101$ and $\mathbf{P t}_{1} \mathbf{C u}_{2} / \mathbf{C}$. Typically, $200 \mathrm{mg}$ of activated MIL-101 powder or graphite carbon was dispersed in $20 \mathrm{~mL}$ of ultrapure water, and was subjected to ultrasonication for $20 \mathrm{~min}$. A fresh $\mathrm{H}_{2} \mathrm{PtCl}_{6} \cdot 6 \mathrm{H}_{2} \mathrm{O}$ and $\mathrm{Cu}$ $\left(\mathrm{NO}_{3}\right)_{2}$ aqueous solution with the desired concentrations were added dropwise to the above solution under vigorous stirring for $\sim 30 \mathrm{~min}$. The suspension was then stirred at room temperature for another $24 \mathrm{~h}$. The impregnated sample was washed with water adequately and was dried under vacuum at $60^{\circ} \mathrm{C}$ for $24 \mathrm{~h}$. The harvested sample was treated in a stream of $20 \% \mathrm{H}_{2} / \mathrm{Ar}\left(40 \mathrm{~mL} \mathrm{~min}^{-1}\right)$ at $200^{\circ} \mathrm{C}$ for $4 \mathrm{~h}$ to yield $\mathrm{Pt}_{1} \mathrm{Cu}_{2} / \mathrm{MIL}-101$ and $\mathrm{Pt}_{1} \mathrm{Cu}_{2} / \mathrm{C}$.

Catalytic performance evaluation for alcohol oxidation. The catalytic reaction was performed in a $10 \mathrm{~mL}$ Teflon-lined stainless-steel autoclave equipped with a pressure gauge and a magnetic stirrer. Upon drying at $120^{\circ} \mathrm{C}$ under vacuum for $12 \mathrm{~h}$, $100 \mathrm{mg}$ of catalyst was dispersed in $5 \mathrm{~mL}$ of water containing $0.2 \mathrm{mmol}$ of alcohol, and the mixture was sonicated for about $20 \mathrm{~min}$ until it became homogeneous. The vessel was then charged with $\mathrm{O}_{2}$ for ten times at room temperature, then pressurized with $\mathrm{O}_{2}$ to $5 \mathrm{bar}$ for reaction. Subsequently, the reaction was conducted at $100^{\circ} \mathrm{C}$ for $5 \mathrm{~h}$ with continuous stirring. After reaction, the catalyst was separated by centrifugation, thoroughly washing with ethanol, and then re-utilized in subsequent runs under identical reaction conditions. The yield of the product was analyzed by GC with an internal standard substance (dodecane).

Catalytic performance evaluation for olefin hydrogenation. In a typical experiment, a mixture of catalyst $(20 \mathrm{mg})$ and olefin $(0.1 \mathrm{mmol})$ was ultrasonically dispersed in a mixture solvent (methanol and water, $\mathrm{V}: \mathrm{V}=5: 1,20 \mathrm{~mL}$ ) or pure solvent (methanol, $20 \mathrm{~mL}$ ) placed in round-bottomed flask $(25 \mathrm{~mL})$. The reduction started when hydrogen source $\left(\mathrm{NH}_{3} \mathrm{BH}_{3}, 30 \mathrm{mg}\right.$ or $\left.\mathrm{NaBH}_{4}, 15 \mathrm{mg}\right)$ was added into the flask. Catalytic yield of olefin hydrogenation reaction was identified by gas chromatography and ${ }^{1} \mathrm{H}$ NMR.

\section{Data availability}

The data that support the findings of this study are available from the corresponding author upon reasonable request.

Received: 16 August 2017 Accepted: 16 July 2019

Published online: 01 August 2019

\section{References}

1. Sheldon, R. A., Arends, I. W. C. E., Brink, G. J. \& Dijksman, A. Green, catalytic oxidations of alcohols. Acc. Chem. Res. 35, 774-781 (2002).

2. Enache, D. I. et al. Solvent-free oxidation of primary alcohols to aldehydes using $\mathrm{Au}-\mathrm{Pd} / \mathrm{TiO}_{2}$ catalysts. Science 311, 362-365 (2006).

3. Zhang, H., Jin, M. \& Xia, Y. Enhancing the catalytic and electrocatalytic properties of Pt-based catalysts by forming bimetallic nanocrystals with Pd. Chem. Soc. Rev. 41, 8035-8049 (2012).

4. Alkilany, A. M., Lohse, S. E. \& Murphy, C. J. The gold standard: gold nanoparticle libraries to understand the nano-bio interface. Acc. Chem. Res. 46, 650-661 (2013).

5. Fan, Z. \& Zhang, H. Template synthesis of noble metal nanocrystals with unusual crystal structures and their catalytic applications. Acc. Chem. Res. 49, 2841-2850 (2016).

6. Bai, L. et al. Explaining the size dependence in platinum-nanoparticlecatalyzed hydrogenation reactions. Angew. Chem. Int. Ed. 55, 15656-15661 (2016).

7. Wu, F. et al. Microfluidic synthesis enables dense and uniform loading of surfactant-free PtSn nanocrystals on carbon supports for enhanced ethanol oxidation. Angew. Chem. Int. Ed. 128, 5036-5040 (2016).

8. Dupont, C., Jugnet, Y. \& Loffreda, D. Theoretical evidence of PtSn alloy efficiency for CO oxidation. J. Am. Chem. Soc. 128, 9129-9136 (2006).

9. Bligaard, T. \& Nørskov, J. K. Ligand effects in heterogeneous catalysis and electrochemistry. Electrochim. Acta 52, 5512-5516 (2007).

10. Tao, F. et al. Reaction-driven restructuring of Rh-Pd and Pt-Pd core-shell nanoparticles. Science 322, 932-934 (2008).

11. Alayoglu, S., Nilekar, A. U., Mavrikakis, M. \& Eichhorn, B. Ru-Pt core-shell nanoparticles for preferential oxidation of carbon monoxide in hydrogen. Nat. Mater. 7, 333-338 (2008).
12. $\mathrm{Xu}, \mathrm{D}$. et al. Solution-based evolution and enhanced methanol oxidation activity of monodisperse platinum-copper nanocubes. Angew. Chem. Int. Ed. 48, 4217-4221 (2009).

13. Jiang, H.-L. \& Xu, Q. Recent progress in synergistic catalysis over heterometallic nanoparticles. J. Mater. Chem. 21, 13705-13725 (2011).

14. He, D. et al. Amorphous nickel boride membrane on a platinum-nickel alloy surface for enhanced oxygen reduction reaction. Nat. Commun. 7, 12632-12639 (2016)

15. Haruta, M. Size- and support-dependency in the catalysis of gold. Catal. Today 36, 153-166 (1997)

16. Campbell, C. T. The energetics of supported metal nanoparticles: relationships to sintering rates and catalytic activity. Acc. Chem. Res. 46, 1712-1719 (2013).

17. Yano, H., Watanable, M., liyama, A. \& Uchida, H. Particle-size effect of Pt cathode catalysts on durability in fuel cells. Nano Energy 29, 323-333 (2016).

18. Yang, C.-m, Kalwei, M., Schüth, F. \& Chao, K.-j Gold nanoparticles in SBA-15 showing catalytic activity in CO oxidation. Appl. Catal. A 254, 289-296 (2003).

19. Zahmakıran, M., Tonbul, Y. \& Özkar, S. Ruthenium(0) nanoclusters stabilized by a nanozeolite framework: isolable, reusable, and green catalyst for the hydrogenation of neat aromatics under mild conditions with the unprecedented catalytic activity and lifetime. J. Am. Chem. Soc. 132, 6541-6549 (2010).

20. Wu, Z., Lv, Y., Xia, Y., Webley, P. A. \& Zhao, D. Ordered mesoporous platinum@graphitic carbon embedded nanophase as a highly active, stable, and methanol-tolerant oxygen reduction electrocatalyst. J. Am. Chem. Soc. 134, 2236-2245 (2012).

21. Tao, F., Zhang, S., Nguyen, L. \& Zhang, X. Action of bimetallic nanocatalysts under reaction conditions and during catalysis: evolution of chemistry from high vacuum conditions to reaction conditions. Chem. Soc. Rev. 41, 7980-7993 (2012)

22. Jiang, C., Hara, K. \& Fukuoka, A. Low-temperature oxidation of ethylene over platinum nanoparticles supported on mesoporous silica. Angew. Chem. Int. Ed. 52, 6265-6268 (2013).

23. Wang, J. et al. Thin porous alumina sheets as supports for stabilizing gold nanoparticles. ACS Nano 7, 4902-4910 (2013).

24. Jiao, L., Seow, J. Y. R., Skinner, W. S., Wang, Z. U. \& Jiang, H.-L. Metalorganic frameworks: structures and functional applications. Mater. Today https://doi.org/10.1016/j.mattod.2018.10.038 (2019).

25. Zhou, H.-C., Long, J. R. \& Yaghi, O. M. Introduction to metal-organic frameworks. Chem. Rev. 112, 673-674 (2012).

26. Zhou, H.-C. \& Kitagawa, S. Metal-organic frameworks (MOFs). Chem. Soc. Rev. 43, 5415-5418 (2014).

27. Cook, T. R., Zheng, Y.-R. \& Stang, P. J. Metal-organic frameworks and selfassembled supramolecular coordination complexes: comparing and contrasting the design, synthesis, and functionality of metal-organic materials Chem. Rev. 113, 734-777 (2013).

28. Zhu, Q.-L. \& Xu, Q. Metal-organic framework composites. Chem. Soc. Rev. 43 5468-5512 (2014).

29. Hu, Z., Deibert, B. J. \& Li, J. Luminescent metal-organic frameworks for chemical sensing and explosive detection. Chem. Soc. Rev. 43, 5815-5840 (2014).

30. Netzer, N. L., Dai, F.-R., Wang, Z. \& Jiang, C. PH-modulated molecular assemblies and surface properties of metal-organic supercontainers at the airwater interface. Angew. Chem. Int. Ed. 53, 10965-10969 (2014).

31. Zeng, L., Guo, X., He, C. \& Duan, C. Metal-organic frameworks: versatile materials for heterogeneous photocatalysis. ACS Catal. 6, 7935-7947 (2016).

32. Li, B. et al. Emerging multifunctional metal-organic framework materials. Adv. Mater. 28, 8819-8860 (2016)

33. Islamoglu, T. et al. Metal-organic frameworks as platforms for functional materials. Acc. Chem. Res. 50, 805-813 (2017).

34. Lu, G. et al. Imparting functionality to a metal-organic framework material by controlled nanoparticle encapsulation. Nat. Chem. 4, 310-316 (2012).

35. Kuo, C. H. et al. Yolk-shell nanocrystal@ZIF-8 nanostructures for gas-phase heterogeneous catalysis with selectivity control. J. Am. Chem. Soc. 134, 14345-14348 (2012)

36. Yang, Q., Xu, Q., Yu, S.-H. \& Jiang, H.-L. Pd Nanocubes@ZIF-8: integration of plasmon-driven photothermal conversion with a metal-organic framework for efficient and selective catalysis. Angew. Chem. Int. Ed. 55, 3685-3689 (2016).

37. Li, Z. et al. Platinum-nickel frame within metal-organic framework fabricated in situ for hydrogen enrichment and molecular sieving. Nat. Commun. 6 , 8248-8256 (2015).

38. Chen, Y.-Z. et al. Singlet oxygen-engaged selective photo-oxidation over Pt nanocrystals/porphyrinic MOF: the roles of photothermal effect and $\mathrm{Pt}$ electronic state. J. Am. Chem. Soc. 139, 2035-2044 (2017).

39. Zhao, M. et al. Metal-organic frameworks as selectivity regulators for hydrogenation reactions. Nature 539, 76-80 (2016). 
40. Hermes, S. et al. Metal@MOF: loading of highly porous coordination polymers host lattices by metal organic chemical vapor deposition. Angew. Chem. Int. Ed. 44, 6237-6241 (2005)

41. Hwang, Y. K. et al. Amine grafting on coordinatively unsaturated metal centers of MOFs: consequences for catalysis and metal encapsulation. Angew. Chem. Int. Ed. 47, 4144-4148 (2008)

42. Jiang, H.-L. et al. Ultrafine gold clusters incorporated into a metal-organic framework. Chem. Eur. J. 17, 78-81 (2011).

43. Aijaz, A. et al. Immobilizing highly catalytically active Pt nanoparticles inside the pores of metal-organic framework: a double solvents approach. J. Am. Chem. Soc. 134, 13926-13929 (2012).

44. Guo, Z. et al. Pt nanoclusters confined within metal-organic framework cavities for chemoselective cinnamaldehyde hydrogenation. ACS Catal. 4, 1340-1348 (2014).

45. Chen, Y.-Z. et al. Multifunctional PdAg@MIL-101 for one-pot cascade reactions: combination of host-guest cooperation and bimetallic synergy in catalysis. ACS Catal. 5, 2062-2069 (2015).

46. Liu, H. et al. Controllable encapsulation of "clean" metal clusters within MOFs through kinetic modulation: towards advanced heterogeneous nanocatalysts. Angew. Chem. Int. Ed. 55, 5019-5023 (2016).

47. Xiao, J.-D. et al. Boosting photocatalytic hydrogen production of a metalorganic framework decorated with platinum nanoparticles: the platinum location matters. Angew. Chem. Int. Ed. 55, 9389-9393 (2016).

48. Yang, Q., Xu, Q. \& Jiang, H.-L. Metal-organic frameworks meet metal nanoparticles: synergistic effect for enhanced catalysis. Chem. Soc. Rev. 46, 4774-4808 (2017).

49. Raftery, D. et al. High-field NMR of adsorbed xenon polarized by laser pumping. Phys. Rev. Lett. 66, 584-587 (1991).

50. Comotti, A., Bracco, S., Valsesia, P., Ferretti, L. \& Sozzani, P. 2D multinuclear NMR, hyperpolarized xenon and gas storage in organosilica nanochannels with crystalline order in the walls. J. Am. Chem. Soc. 129, 8566-8576 (2007).

51. Liu, Y. et al. Probing the porosity of cocrystallized MCM-49/ZSM-35 zeolites by hyperpolarized ${ }^{129}$ Xe NMR. J. Phys. Chem. B 112, 1226-1231 (2008).

52. Cheng, C.-Y., Stamatatos, T. C., Christou, G. \& Bowers, C. R. Molecular wheels as nanoporous materials: differing modes of gas diffusion through $\mathrm{Ga}_{10}$ and $\mathrm{Ga}_{18}$ wheels probed by hyperpolarized ${ }^{129}$ Xe NMR spectroscopy. J. Am. Chem. Soc. 132, 5387-5393 (2010).

53. Huang, J. et al. Propene epoxidation with $\mathrm{O}_{2}$ and $\mathrm{H}_{2}$ : identification of the most active gold clusters. J. Catal. 278, 8-15 (2011).

54. Böhlmann, W., Pöppl, A., Sabo, M. \& Kaskel, S. Characterization of the metalorganic framework compound $\mathrm{Cu}_{3}$ (benzene $1,3,5$-tricarboxylate) ${ }_{2}$ by means of ${ }^{129} \mathrm{Xe}$ nuclear magnetic and electron paramagnetic resonance spectroscopy. $J$. Phys. Chem. B 110, 20177-20181 (2006).

55. Comotti, A. et al. Nanochannels of two distinct cross-sections in a porous Albased coordination polymer. J. Am. Chem. Soc. 130, 13664-13672 (2008).

56. Springuel-Huet, M.-A. et al. ${ }^{129}$ Xe NMR study of the framework flexibility of the porous hybrid MIL-53(Al). J. Am. Chem. Soc. 132, 11599-11607 (2010).

57. Hoffmann, H. C. et al. High-pressure in situ ${ }^{129}$ Xe NMR spectroscopy and computer simulations of breathing transitions in the metal-organic framework $\mathrm{Ni}_{2}(2,6-\mathrm{ndc})_{2}$ (dabco) (DUT-8(Ni)). J. Am. Chem. Soc. 133, 8681-8690 (2011).

58. He, C. et al. Tunable pores in mesoporous silica films studied using a pulsed slow positron beam. Radiat. Phys. Chem. 76, 204-208 (2007).

59. Guan, M. et al. Vacancy associates promoting solar-driven photocatalytic activity of ultrathin bismuth oxychloride nanosheets. J. Am. Chem. Soc. 135, 10411-10417 (2013).

60. Petzetakis, N. et al. Membranes with artificial free-volume for biofuel production. Nat. Commun. 6, 7529-7536 (2015).

61. Zhang, H. J. et al. Charge-to-spin conversion and spin diffusion in $\mathrm{Bi} / \mathrm{Ag}$ bilayers observed by spin-polarized positron beam. Phys. Rev. Lett. 114, 166602 (2015). (1-5).

62. Férey, G. et al. A chromium terephthalate-based solid with unusually large pore volumes and surface area. Science 309, 2040-2042 (2005).

63. Kansy, J. Microcomputer program for analysis of positron annihilation lifetime spectra. Nucl. Instrum. Methods Phys. Res. A 374, 235-244 (1996).
64. Kong, M. et al. Tuning the relative concentration ratio of bulk defects to surface defects in $\mathrm{TiO}_{2}$ nanocrystals leads to high photocatalytic efficiency. J. Am. Chem. Soc. 133, 16414-16417 (2011).

65. Chakraverty, S., Mitra, S., Mandal, K., Nambissan, P. M. G. \& Chattopadhyay, S. Positron annihilation studies of some anomalous features of $\mathrm{NiFe}_{2} \mathrm{O}_{4}$ nanocrystals grown in $\mathrm{SiO}_{2}$. Phys. Rev. B. 71, 024115 (2005).

66. Puska, M. J., Lanki, P. \& Nieminen, R. M. Positron affinities for elemental metals. J. Phys.: Condens. Matter 1, 6081 (1989).

67. Nagai, Y. et al. Positron confinement in ultrafine embedded particles: quantum-dot-like state in an Fe-Cu alloy. Phys. Rev. B. 61, 6574 (2000).

68. Dutta, D. et al. Evidence of positronium bloch states in porous crystals of $\mathrm{Zn}_{4} \mathrm{O}$-coordination polymers. Phys. Rev. Lett. 110, 197403 (2013).

69. Crivelli, P. et al. Positronium emission spectra from self-assembled metalorganic frameworks. Phys. Rev. B 89, 241103 (2014).

70. Nandi, S. et al. A single-ligand ultra-microporous MOF for precombustion $\mathrm{CO}_{2}$ capture and hydrogen purification. Sci. Adv. 1, e1500421 (2015).

\section{Acknowledgements}

This work was supported by the NSFC (21725101, 21871244, 21673213, 21521001, and 21701093), the NSF of Shandong Province (ZR2017BB044), China Postdoctoral Science Fund (2018T110664 and 2017M622127), Fujian Institute of Innovation (CAS) and Key Research and Development Program of Shandong Province (2019GGX103043).

\section{Author contributions}

H.-L.J. conceived the idea and supervised the project. Y.-Z.C. designed and performed the experiments and collected the data. B.G. performed the positron annihilation experiment and analyzed the results. T.U. performed the tomography and slice measurements. X.L. performed the hyperpolarized ${ }^{129}$ Xe NMR measurements. H.-L.J. and Y.-Z.C. analyzed the data and co-wrote the paper. T.U., J.L., X.L., B.-J.Y., and Q.X. discussed the results and commented the paper.

\section{Additional information}

Supplementary Information accompanies this paper at https://doi.org/10.1038/s41467019-11449-6.

Competing interests: The authors declare no competing interests.

Reprints and permission information is available online at http://npg.nature.com/ reprintsandpermissions/

Peer review information: Nature Communications thanks the anonymous reviewers for their contribution to the peer review of this work.

Publisher's note: Springer Nature remains neutral with regard to jurisdictional claims in published maps and institutional affiliations.

Open Access This article is licensed under a Creative Commons Attribution 4.0 International License, which permits use, sharing, adaptation, distribution and reproduction in any medium or format, as long as you give appropriate credit to the original author(s) and the source, provide a link to the Creative Commons license, and indicate if changes were made. The images or other third party material in this article are included in the article's Creative Commons license, unless indicated otherwise in a credit line to the material. If material is not included in the article's Creative Commons license and your intended use is not permitted by statutory regulation or exceeds the permitted use, you will need to obtain permission directly from the copyright holder. To view a copy of this license, visit http://creativecommons.org/ licenses/by/4.0\%.

(C) The Author(s) 2019 